\title{
The value of intraoperative MRI in recurrent intracranial tumor surgery
}

\author{
Sophie S. Wang, MD,, ${ }^{1,2}$ Friederike Selge, MD, ${ }^{2}$ Martina Sebök, MD, ${ }^{3}$ Pierre Scheffler, MD, ${ }^{3}$ \\ Yang Yang, MD, ${ }^{3,5}$ Giovanna Brandi, MD, ${ }^{2}$ Sebastian Winklhofer, MD, ${ }^{4}$ and Oliver Bozinov, MD ${ }^{3,5}$

\begin{abstract}
1Department of Neurosurgery, Eberhard Karls University Tübingen, Germany; ${ }^{2}$ Institute of Intensive Care Medicine, University Hospital Zurich; ${ }^{3}$ Department of Neurosurgery, University Hospital Zurich, Clinical Neuroscience Center, University of Zurich; ${ }^{4}$ Department of Neuroradiology, University Hospital Zurich, Clinical Neuroscience Center, Zurich; and ${ }^{5}$ Department of Neurosurgery, Kantonsspital St. Gallen, Medical School St. Gallen, Switzerland
\end{abstract}

\begin{abstract}
OBJECTIVE Identifying tumor remnants in previously operated tumor lesions remains a challenge. Intraoperative MRI (ioMRI) helps the neurosurgeon to reorient and update image guidance during surgery. The purpose of this study was to analyze whether ioMRI is more efficient in detecting tumor remnants in the surgery of recurrent lesions compared with primary surgery.

METHODS All consecutive patients undergoing elective intracranial tumor surgery between 2013 and 2018 at the authors' institution were included in this retrospective cohort study. The cohort was divided into two groups: re-craniotomy and primary craniotomy. In contrast-enhancing tumors, tumor suspicion in ioMRI was defined as contrast enhancement in T1-weighted imaging. In non-contrast-enhancing tumors, tumor suspicion was defined as hypointensity in T1weighted imaging and hyperintensity in T2-weighted imaging and FLAIR. In cases in which the ioMRI tumor suspicion was a false positive and not confirmed during in situ inspection by the neurosurgeon, the signal was defined as a tumorimitating ioMRI signal (TIM). Descriptive statistics were performed.
\end{abstract}

RESULTS A total of 214 tumor surgeries met the inclusion criteria. The re-craniotomy group included 89 surgeries, and the primary craniotomy group included 123 surgeries. Initial complete resection after ioMRI was less frequent in the recraniotomy group than in the primary craniotomy group, but this was not a statistically significant difference. Radiological suspicion of tumor remnants in ioMRI was present in $78 \%$ of re-craniotomy surgeries and $69 \%$ of primary craniotomy surgeries. The incidence of false-positive TIMs was significantly higher in the re-craniotomy group $(n=11,12 \%)$ compared with the primary craniotomy group $(n=5,4 \% ; p=0.015)$, and in contrast-enhancing tumors was related to hemorrhages in situ $(n=9)$.

CONCLUSIONS A history of previous surgery in contrast-enhancing tumors made correct identification of tumor remnants in ioMRI more difficult, with a higher rate of false-positive ioMRI signals in the re-craniotomy group. The majority of TIMs were associated with the inability to distinguish contrast enhancement from hyperacute hemorrhage. The addition of a specific sequence in ioMRI to further differentiate both should be investigated in future studies.

https://thejns.org/doi/abs/10.3171/2020.6.JNS20982

KEYWORDS intraoperative magnetic resonance imaging; neurooncology; recurrence; oncology

$\mathrm{G}$ ROSS-TOTAL resection (GTR) has been associated with increased survival in several recurrent brain tumor entities..$^{1-5}$ Conventional neuronavigation based on preoperative MRI provides limited reliability in the course of surgery. ${ }^{6-8}$ By performing intraoperative MRI (ioMRI), it is possible to update neuronavigation, compensate for brain shift, and help achieve maximal re- section in a timely manner. ${ }^{9-11}$ In modern neurosurgery, ioMRI is an important factor to be considered in intraoperative decision-making.

Gliosis, scarring, posttherapeutic rigidity, and radionecrosis influence tissue characteristics such as texture, elasticity, or vasculature. ${ }^{12}$ Therefore, during surgery of recurrent lesions, anatomical orientation in the brain pa-

ABBREVIATIONS 5-ALA = 5-aminolevulinic acid; DWI = diffusion-weighted imaging; EOR = extent of resection; GTR = gross-total resection; ioMRI = intraoperative MRI; iONM = intraoperative neuromonitoring; ioUS = intraoperative ultrasound; SWI = susceptibility weighted imaging; TIM = tumor-imitating ioMRI signal.

SUBMITTED March 29, 2020. ACCEPTED June 1, 2020.

INCLUDE WHEN CITING Published online October 2, 2020; DOI: 10.3171/2020.6.JNS20982. 
renchyma can be more difficult than in cases without prior surgery. In recurrent gliomas, 5-aminolevulinic acid (5-ALA) fluorescence has shown false-positive results. ${ }^{12}$ Intraoperative ultrasound (ioUS) is widely used, but its sensitivity has been reported to be inferior in recurrent tumors compared with newly diagnosed ones. ${ }^{13}$ Thus, finding tumor remnants in recurrent lesions remains a challenge. The particular purpose of this study is to evaluate the value of ioMRI as an intraoperative modality in the surgery of recurrent lesions compared with newly diagnosed lesions.

\section{Methods \\ Study Design}

This retrospective blinded cohort study analyzed all consecutive patients undergoing elective intracranial tumor surgery between 2013 and 2018 at the Department of Neurosurgery, University Hospital Zurich. The study population was identified through a prospective registry. The local ethics committee approved data collection and post hoc analysis.

\section{Study Group}

Inclusion criteria were complete data on intraoperative decision-making and elective craniotomy for tumor extirpation using ioMRI. Exclusion criteria were procedures with only burr holes, e.g., stereotactic biopsies or transnasal tumor resection. The cohort was divided into two groups: those who had recurrent lesions with a known history of neurosurgical tumor resection were classified in the re-craniotomy group, and those who had no previous surgeries were classified in the primary craniotomy group.

\section{Intraoperative MRI at the Study Institution}

The ioMRI at our study institution has been installed in a two-room setup, in which the patient is draped specifically to be transferred from a sterile to a nonsterile environment, where the high-field 3-T MRI suite is located. ${ }^{14}$ Temporary closure of the incision consists of a gross dural closure and fixation of the cutaneous and subcutaneous layers. The patient is then specially draped for transfer to the MRI suite. ${ }^{14}$ The MRI sequences were acquired based on our institutional guidelines. These sequences consist of T1-weighted, T2-weighted, FLAIR, and gadoliniumenhanced T1-weighted sequences and diffusion-weighted imaging (DWI). For reestablishment of the surgical field, the patient is undraped and then sterile-draped again for surgery.

\section{Intraoperative Decision-Making and Finding}

In cases of tumor suspicion on ioMRI, the leading neurosurgeon made the decision on whether to reopen the operative field. In contrast-enhancing tumors, tumor suspicion in ioMRI was defined as contrast enhancement in T1-weighted imaging. In non-contrast-enhancing tumors, tumor suspicion was defined as hypointensity in T1-weighted imaging and hyperintensity in T2-weighted imaging and FLAIR. The consecutive in situ finding was described by the neurosurgeon, and the operative report was dictated right after surgery. Intraoperative MRI findings were divided according to the neurosurgeon's finding intraoperatively (Fig. 1). In cases in which the ioMRI tumor suspicion was a false positive and not confirmed during in situ inspection by the neurosurgeon, the signal was defined as a tumor-imitating ioMRI signal (TIM).

\section{Data Collection}

The study population was identified through a prospective registry. Patient demographics (age and gender), tumor characteristics (localization, pathology, and WHO grade), extent of resection (EOR), postoperative ischemia, medical history (previous operation and radio- or chemotherapy), neuroradiological report on imaging, and surgery characteristics (ultrasound, neuromonitoring, and fluorescence guidance) were analyzed blinded and retrospectively. EOR and postoperative ischemia (in DWI) at the end of surgery were evaluated either in ioMRI, if no additional resection was performed, or in early postoperative MRI (24-72 hours after surgery). Tumor location was classified into frontal, temporal, parietal, occipital, insular, brainstem, and cerebellar.

\section{Statistical Analysis}

Statistical analysis was performed in R Studio using descriptive statistics. Significance was defined as the probability of a two-sided type 1 error being $<5 \%$ ( $p<$ $0.05)$. To compare nonnumeric parameters of both groups, the chi-square test was applied. For numeric parameters, Welch's two-sample t-test was applied.

\section{Results \\ Patient Population}

In total, 214 tumor surgeries on 184 patients met the inclusion criteria. Of them, 89 (42\%) were cases of recraniotomy, while 125 cases $(58 \%)$ were in the primary craniotomy group. Patient demographics are shown in Table 1 . In the re-craniotomy group, 56 patients $(63 \%)$ had received prior chemotherapy $(n=6)$, radiotherapy $(n=12)$, or both $(n=38)$.

\section{Lesion Characteristics}

Eighty percent of the tumors were supratentorial. In the whole cohort, 55 cases $(26 \%)$ were non-contrast-enhancing tumors, and the remaining 159 cases (74\%) were gadolinium contrast-enhancing on T1-weighted MRI. Anatomical characteristics are shown in Table 1.

\section{Other Intraoperative Tools and OR Time}

The usage of intraoperative neuromonitoring (ioNM), ioUS, image-guided neuronavigation, and 5-ALA in both groups is shown in Tables 2 and 3. The rate of ioUS utilization in the study group was significantly lower $(\mathrm{p}=$ 0.003). The mean operating duration in the re-craniotomy group was significantly lower compared with the control group ( $\mathrm{p}<0.001$; Table 3$)$.

\section{ioMRI and Its Consequential Decision-Making}

No patient underwent more than one ioMRI. Initial 


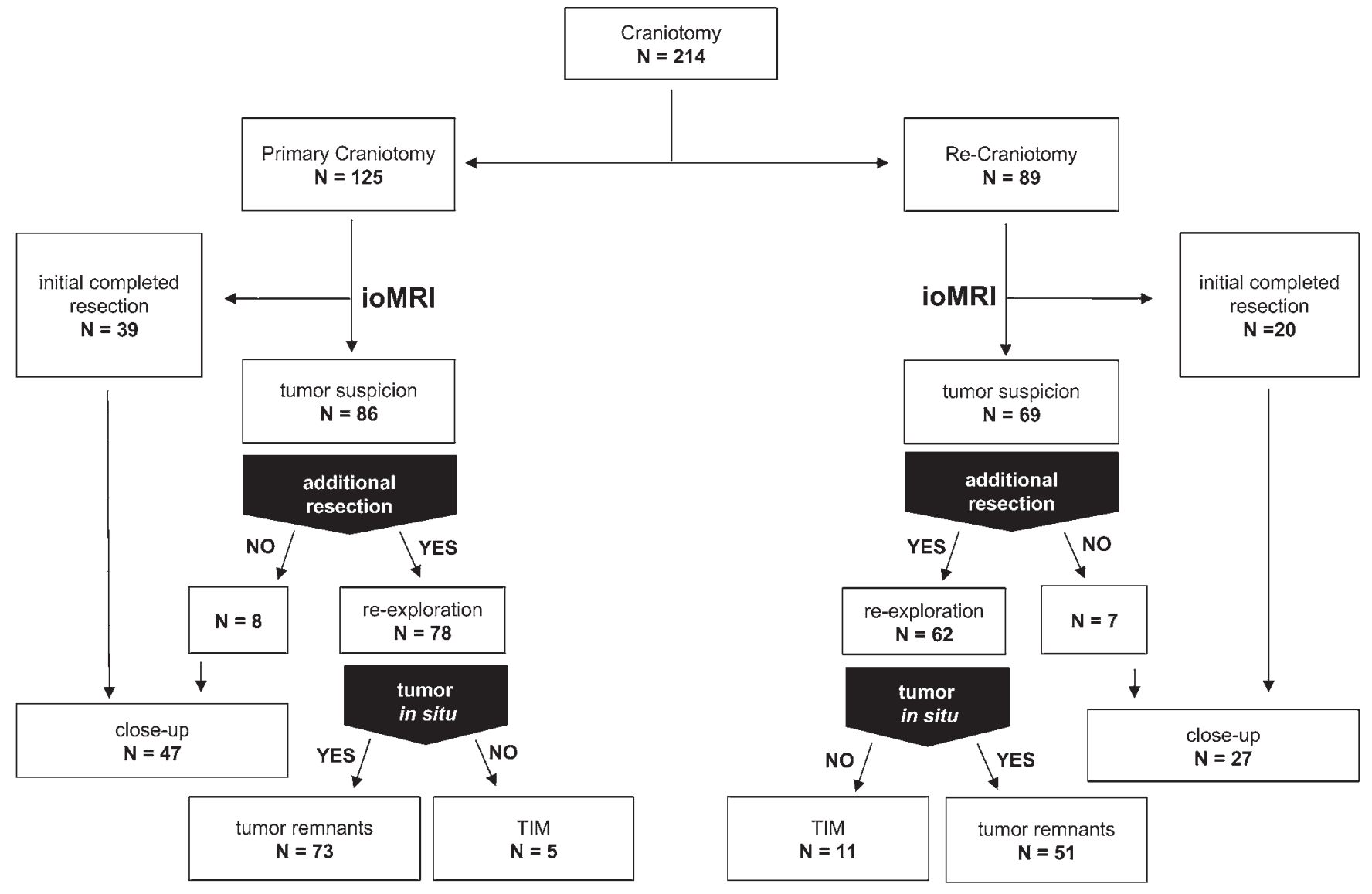

FIG. 1. Flowchart of ioMRI decision-making.

complete resection confirmed by ioMRI was achieved in 20 of 89 re-craniotomy operations (22\%). In $78 \%$ of surgeries in the re-craniotomy group, tumor suspicion was raised after ioMRI. In primary craniotomy, 39 patients (31\%) showed complete resection in ioMRI, and 86 of the 125 ioMRI results (69\%) showed tumor suspicion. Even though initial complete resection was less frequent in recraniotomy than in primary craniotomy, this value showed no statistical significance $(p=0.185)$. In 8 cases of primary craniotomy and 7 cases in re-craniotomy, the neurosurgeon deliberately did not further resect due to planned partial resection (e.g., due to residual tumor in eloquent area). In all other cases of tumor suspicion in ioMRI, reexploration was performed and the in situ finding evaluated; the result was the phenomenon of TIM in 11 cases (12\%) of re-craniotomy and 5 cases (4\%) of primary craniotomy. The described TIMs turned out to be a hemorrhage, fibrosis (5-ALA nonfluorescent in 5-ALA-positive tumors), dura, Tabotamp (a hemostatic agent), plexus, or vessels in situ (Table 2, Fig. 2). The incidence of TIM was significantly higher in the re-craniotomy group $(\mathrm{p}=0.015$; Table 3 ) and was related to hemorrhages in situ $(n=9,60 \%)$. The rate of TIM was not significantly increased in either the chemotherapy $(n=42)$ or radiotherapy $(n=46)$ group $(p=$ 0.383 and $\mathrm{p}=0.861$, respectively).

One TIM was found during surgery for a non-contrastenhancing oligoastrocytoma (Fig. 2B). All other TIMs $(\mathrm{n}=15)$ were contrast enhancement signals in contrastenhancing tumors. Analysis of the incidence of TIM in contrast-enhancing versus non-contrast-enhancing settings showed that there was no statistically significant difference between the two $(p=0.096)$. The rate of reexploration and EOR in contrast-enhancing versus non-contrast-enhancing settings showed no significant difference ( $\mathrm{p}=0.661$ and $\mathrm{p}=0.885$, respectively).

GTR was achieved with the help of ioMRI in 72 of all primary craniotomy cases (58\%) and 55 of all re-craniotomy cases $(62 \% ; \mathrm{p}=0.542)$. Postoperative ischemia on DWI was found with a similar incidence in 27 cases $(22 \%)$ in the primary craniotomy group and 17 cases (19\%) in the re-craniotomy group $(\mathrm{p}=0.829)$.

\section{Discussion}

\section{ioMRI and Recurrent Surgery}

To the best of our knowledge, this is the first cohort study designed to specifically compare parameters related to and affecting intraoperative decision-making based on ioMRI in a re-craniotomy versus primary craniotomy setting. As a consequence of our study design and objective, the number of recurrent tumor cases included in our analysis is larger than those in existing studies (Table 4). No significant association with additional resection after ioMRI in recurrent lesions was reported in the literature, which is consistent with our study results. ${ }^{15}$ 
TABLE 1. Patient demographics and tumor characteristics

\begin{tabular}{|c|c|c|c|}
\hline Characteristic & All & Primary Craniotomy & Re-Craniotomy \\
\hline No. of operations & 214 & 125 & 89 \\
\hline Mean age \pm SD, yrs & $39.9 \pm 20.8$ & $40.1 \pm 21.3$ & $39.6 \pm 19.6$ \\
\hline Males & $133(62)$ & $77(62)$ & $56(63)$ \\
\hline Females & $81(38)$ & $48(38)$ & $33(37)$ \\
\hline \multicolumn{4}{|l|}{ Tumor type } \\
\hline Ependymoma & $13(6)$ & $6(5)$ & $7(8)$ \\
\hline Low-grade glioma (WHO grade I-II) & $44(20)$ & $26(21)$ & $18(20)$ \\
\hline High-grade glioma (WHO grade III-IV) & $116(54)$ & $70(56)$ & $45(51)$ \\
\hline Medulloblastoma & $10(5)$ & $8(6)$ & $2(2)$ \\
\hline Meningioma & $2(1)$ & $1(1)$ & $1(1)$ \\
\hline Metastatic & $13(6)$ & $6(5)$ & $7(8)$ \\
\hline Schwannoma & $2(1)$ & $1(1)$ & $1(1)$ \\
\hline Other & $14(7)$ & $7(6)$ & $7(8)$ \\
\hline \multicolumn{4}{|l|}{ Tumor location } \\
\hline CPA & $6(3)$ & $2(2)$ & $4(4)$ \\
\hline Frontal & $78(36)$ & $43(34)$ & $35(39)$ \\
\hline Insular & $15(7)$ & $9(7)$ & $6(7)$ \\
\hline Occipital & $8(4)$ & $6(5)$ & $2(2)$ \\
\hline Parietal & $27(13)$ & $15(12)$ & $12(13)$ \\
\hline Thalamic & $5(2)$ & $4(3)$ & $1(1)$ \\
\hline Temporal & $24(11)$ & $17(14)$ & $7(8)$ \\
\hline Central & $16(7)$ & $7(6)$ & $9(10)$ \\
\hline Cerebellar & $10(5)$ & $5(4)$ & $5(6)$ \\
\hline Brainstem & $4(2)$ & $2(2)$ & $2(2)$ \\
\hline Intraventricular & $15(7)$ & $11(9)$ & $4(4)$ \\
\hline Other & $6(3)$ & $4(3)$ & $2(2)$ \\
\hline \multicolumn{4}{|l|}{ Contrast enhancement } \\
\hline Yes & $159(74)$ & $87(70)$ & $72(81)$ \\
\hline No & $55(26)$ & $38(30)$ & $17(19)$ \\
\hline
\end{tabular}

In a recurrent setting, neurooncological care has developed toward finding molecular classifiers for personalized medicine. ${ }^{16}$ Thus, reoperation is important for tumor volume reduction and choice of adjuvant therapy. ${ }^{1,16}$ In contrast with the focus of neurooncological research on recurrent lesions (recruitment of patients with a recurrence in clinical trials), neurosurgical studies mostly focus on primary lesions. Thus, we lack a clear recommendation on how to technically operate on recurrent intracranial tumors. The same applies to the relevance of ioMRI: it has been studied extensively in primary disease, but only a few studies include recurrent disease. .,6, $, 15,17-22^{2}$

\section{The Definition of TIM}

To our knowledge, this is the first analysis to describe TIM in the context of ioMRI-guided tumor surgery. All TIMs were reported in intrinsic brain tumors, and no TIMs were observed in metastasis surgery. Due to the lack of association of TIM with a history of radio- or chemo- therapy, we postulate that the increased phenomenon of TIM in the re-craniotomy group was not a direct result of adjuvant therapy (chemo- or radiotherapy) and its effect on the parenchyma, but instead a result of the changes in tissue following the trauma of surgery.

The incidence of TIM in our series was highly associated with gadolinium-based contrast enhancement (15 of 16 cases). The majority of TIMs in contrast-enhancing tumors $(>60 \%)$ in the ioMRI were related to hematoma in the operating field. Identification of hyperacute intraparenchymal hemorrhage has proven to be very challenging in T1- or T2-weighted MR images. ${ }^{23}$ In intraoperative decision-making, the timeframe of hemorrhage is usually less than 6 hours and, therefore, too early for either of those sequences. However, MRI offers additional hemosiderinsensitive sequences, such as $\mathrm{T} 2 *$-weighted gradient echo sequences or susceptibility weighted imaging (SWI). The latter would be a highly sensitive sequence for hyperacute deoxygenated hemoglobin and could be used to distin- 
TABLE 2. Tumor-imitating ioMRI signal

\begin{tabular}{|c|c|c|c|c|c|c|c|c|c|}
\hline $\begin{array}{l}\text { TIM } \\
\text { No. }\end{array}$ & Surgery & $\begin{array}{c}\text { TIM } \\
\text { Finding }\end{array}$ & $5-A L A$ & $\begin{array}{c}\text { Gd } \\
\text { Contrast }\end{array}$ & EOR & Localization & $\begin{array}{l}\text { OR Time } \\
\text { (mins) }\end{array}$ & Pathology & $\begin{array}{l}\text { WHO } \\
\text { Grade }\end{array}$ \\
\hline 1 & PC & Hemorrhage \& hemostatic agent & No & Yes & GTR & Brainstem & 380 & Astrocytoma & I \\
\hline 2 & PC & Arachnoid cyst & No & Yes & GTR & CPA & 317 & Ependymoma & III \\
\hline 3 & PC & Hemorrhage & Yes & Yes & PR & Frontal & 295 & Glioma & IV \\
\hline 4 & PC & Hemorrhage & Yes & Yes & PR & Temporal & 300 & Glioma & IV \\
\hline 5 & PC & Plexus & No & Yes & GTR & Intraventricular & 305 & Medulloblastoma & IV \\
\hline 6 & $\mathrm{RC}$ & Hemorrhage & No & Yes & GTR & Thalamic & 330 & Astrocytoma & 1 \\
\hline 7 & $\mathrm{RC}$ & Hemorrhage & No & No & GTR & Temporal & 495 & Astrocytoma & II \\
\hline 8 & $\mathrm{RC}$ & Granulation & No & Yes & GTR & Frontal & 285 & Astrocytoma & III \\
\hline 9 & $\mathrm{RC}$ & Hemorrhage & No & Yes & GTR & CPA & 220 & Astrocytoma & III \\
\hline 10 & $\mathrm{RC}$ & Granulation & No & Yes & PR & Intraventricular & 390 & Central neurocytoma & $\|$ \\
\hline 11 & $\mathrm{RC}$ & Dura & No & Yes & GTR & Central & 255 & Ependymoma & III \\
\hline 12 & $\mathrm{RC}$ & Hemorrhage & Yes & Yes & PR & Frontal & 225 & Glioma & III \\
\hline 13 & $\mathrm{RC}$ & Granulation & Yes & Yes & GTR & Parietal & 282 & Glioma & IV \\
\hline 14 & $\mathrm{RC}$ & Hemorrhage & No & Yes & GTR & Frontal & 150 & Metastasis & NA \\
\hline 15 & $\mathrm{RC}$ & Hemorrhage \& hemostatic agent & No & Yes & GTR & Frontal & 270 & Myxoma & NA \\
\hline 16 & $\mathrm{RC}$ & Vessel & Yes & Yes & GTR & Parietal & 425 & Glioma & IV \\
\hline
\end{tabular}

$\mathrm{NA}=$ not applicable; $\mathrm{OR}$ time = operating time; $\mathrm{PC}=$ primary craniotomy $\mathrm{PR}=$ partial resection; $\mathrm{RC}=$ re-craniotomy .

guish contrast enhancement between early hemorrhage and residual tumor in ioMRI. ${ }^{24}$ SWI sequences were not part of our original ioMRI protocol, and we could not find any previous data on the usage of SWI or similar sequences during ioMRI in the resection of brain tumors. Similarly to our institutional guidelines, most previous studies on ioMRI define residual tumor as contrast enhancement on T1-weighted imaging. ${ }^{6}$ SWI gradient-recalled echo sequences are able to reliably detect hematoma as early as 10 minutes after injection in animal models. ${ }^{25}$ The evaluation of sequences such as SWI to differentiate residual tumor and hemorrhage in ioMRI should be investigated.

Other radiological features that can be used to differ-

TABLE 3. Primary craniotomy versus re-craniotomy

\begin{tabular}{|c|c|c|c|}
\hline Parameter & $\begin{array}{c}\text { Primary } \\
\text { Craniotomy }\end{array}$ & Re-Craniotomy & $\begin{array}{c}p \\
\text { Value }\end{array}$ \\
\hline No. of operations & 125 & 89 & \\
\hline Mean age $\pm S D$, yrs & $41.1 \pm 21.1$ & $38.1 \pm 20.2$ & 0.291 \\
\hline Neuronavigation & $118(94)$ & $84(94)$ & 0.983 \\
\hline ioNM & $73(58)$ & $45(51)$ & 0.326 \\
\hline 5-ALA & $23(18)$ & $24(27)$ & 0.464 \\
\hline ioUS & $102(82)$ & $58(65)$ & 0.003 \\
\hline Mean op time $\pm S D$, mins & $359.22 \pm 108.26$ & $312.43 \pm 89.75$ & $<0.001$ \\
\hline Postop ischemia & $27(22)$ & $17(19)$ & 0.829 \\
\hline Initial complete resection & $39(31)$ & $20(22)$ & 0.574 \\
\hline GTR & $72(58)$ & $55(62)$ & 0.542 \\
\hline Complications & $3(2)$ & $2(2)$ & 0.959 \\
\hline TIM & $5(4)$ & $11(12)$ & 0.015 \\
\hline
\end{tabular}

Values are presented as the number of patients (\%) unless indicated otherwise. Boldface type indicates statistical significance. entiate TIM from residual tumor include the morphology of the suspected abnormality, such as nodular or linear patterns of contrast enhancement, as well as additional advanced MRI techniques, such as DWI or perfusionweighted imaging. DWI allows for an assessment of the cellularity of the abnormality, whereas perfusion-weighted imaging allows one to investigate the vascularization of the lesion. However, as the area of interest is often quite small, both techniques might be of limited value because reliable qualitative or quantitative region of interest analyses might not be applicable.

Additionally, our findings pose the question of whether brain parenchyma that has been treated by prior surgery, chemotherapy, or radiotherapy is more prone to hemorrhage and therefore to hemorrhage-related TIM.

\section{Limitations and Strengths of the Study}

It is apparent that the retrospective nature of this study bears its limitations and biases. We were able to find an association of TIM with recurrent tumor surgery; however, its definite causal verification and prevalence could only be truly understood by a prospective design with systematic histopathological characterization of TIM. Knowledge of why and when the surgeon decides to perform ioMRI (confirmation of GTR, update of navigation, or localization of tumor remnants) would also be valuable. ${ }^{26}$ We were not able to reliably collect these data retrospectively. Due to the low incidence of TIM in non-contrastenhancing tumors $(\mathrm{n}=1)$, we were not able to associate it with a factor (such as hemorrhage), as we have done with contrast-enhancing TIM. The phenomenon of TIM in non-contrast-enhancing tumors should therefore be investigated on a larger scale to understand it better.

However, very detailed and excellently kept electronic medical records-including neuroradiological reports and 

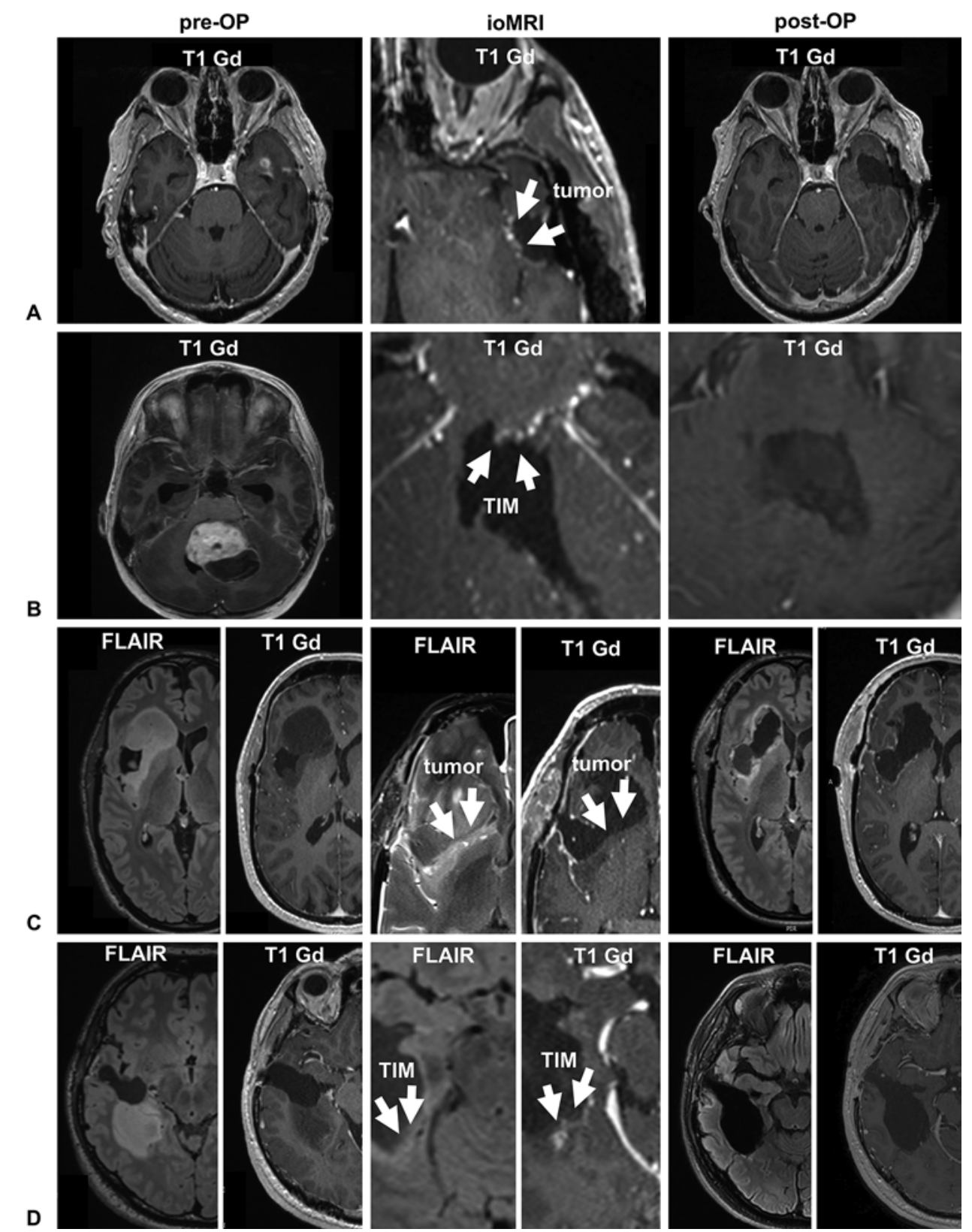

FIG. 2. Images showing the phenomenon of TIM. A: Intraoperative MRI shows residual tumor of a contrast-enhancing glioblastoma (WHO grade IV) in the left medial temporal gyrus in a 62-year-old woman (re-craniotomy). A year previously, she underwent her first operation with ioMRI, resulting in a complete resection. She had received radio- and chemotherapy (temozolomide). During therapy, she presented with this new contrast-enhancing tumor (see preoperative image). Reoperation was performed by reopening the temporal craniotomy. Intraoperative NM and 5-ALA were used to help guide resection. Contrast enhancement (arrows) was visible in the axial ioMRI, which in situ showed 5-ALA fluorescence and histopathologically confirmed pleomorphic, astrocytic tumor cells. B: Intraoperative MRI showed TIM in a contrast-enhancing tumor in a case of a 9-year-old girl (primary craniotomy). The tumor was located in the fourth ventricle. Surgery was performed through a suboccipital craniotomy. The axial ioMRI showed contrast enhancement (arrows). Reopening of the operative field showed no tumor remnant, but Tabotamp and a small hematoma. The Tabotamp was exchanged intraoperatively and hemostasis performed. An additional postoperative MR image confirmed complete resection, and the child remains radiologically tumor free today. The pathology report for the tumor stated it was a pilocytic astrocytoma (WHO grade I). C: Intraoperative MRI showed residual tumor in a contrast-enhancing tumor of a 21-year-old man with recurrent insular astrocytoma (WHO grade II; re-craniotomy). Primary surgery was performed a year previously without ioMRI but with ioUS and intraoperative navigation. Only partial resection was achieved. The patient now presented with progression of the residual tumor. This reoperation was performed using ioMRI, which showed FLAIR hyperintensity and T1-weighted hypointensity craniolaterally and near the basal ganglia (arrows). The neurosurgeon resected residual tumor until he visualized the putamen. The postoperative images show partial resection. The patient received adjuvant proton radiation therapy and systemic treatment. D: Intraoperative MRI shows TIM in a non-contrast-enhancing tumor of a 32-year-old man who was operated on using ioMRI (re-craniotomy). He had a known diffuse oligoastrocytoma (WHO grade II), which was resected completely just 1 year previously. The lesion was temporal, and reoperation was performed by enlargement of the old frontotemporal craniotomy. Axial ioMRI showed hyperintensity on FLAIR and T1-weighted contrast enhancement (arrows). Tumor progression with contrast enhancement could not be ruled out; therefore, reinspection was initiated. In situ inspection showed a small hematoma. Pre- and postoperative blood workup for coagulation were inconspicuous. 
TABLE 4. Number of cases of recurrent intracranial tumor surgeries included in studies on ioMRI

\begin{tabular}{|c|c|c|}
\hline Authors \& Year & Total Cohort & Recurrent Cohort \\
\hline Bohinski et al., $2001^{15}$ & 50 & 21 \\
\hline Nimsky et al., $2004^{17}$ & 47 & 10 \\
\hline Hatiboglu et al., $2009^{18}$ & 46 & 20 \\
\hline Kuhnt et al., $2011^{19}$ & 135 & 27 \\
\hline Coburger et al., $2014^{20}$ & 45 & 7 \\
\hline Quick-Weller et al., $2016^{22}$ & 8 & 8 \\
\hline Coburger et al., $2016^{21}$ & 288 & 49 \\
\hline Scherer et al., $2020^{3}$ & 140 & 30 \\
\hline Current study & 214 & 89 \\
\hline
\end{tabular}

Values are presented as the number of patients.

a detailed operative report requested from every leading neurosurgeon to be dictated right after the operationhelped with the retrospective analysis as well as with comprehending the correlation of MRI interpretation and findings in situ. Moreover, we managed to compare our study group (re-craniotomy) with a control group (primary craniotomy), which was treated in an identical manner (ioMRI protocol, surgeons, neuronavigation, use of ioUS, etc.). In our opinion, this retrospective analysis plays a pivotal role in uncovering an unknown association of increased TIM in surgery to recurrent surgeries, which can now be explored further in future studies.

\section{Future Directions and Study Significance}

Intraoperative MRI is not yet widely available. We believe that it is of even greater importance to make an effort to determine the value of ioMRI and its influence on intraoperative decision-making for those neurosurgical institutions that are thinking about installing ioMRI. The identification of TIM demonstrates that hyperacute changes from surgery performed only several hours to minutes before the ioMRI and their concordant MRI signals can be more difficult to interpret than conventional diagnostic MRI (especially in recurrent lesions). This topic needs further investigation, especially in the field of correlation of imaging properties to histopathological and fluorescence findings in ioMRI-guided surgery.

Our findings do not diminish the value of ioMRI in the reoperation setting. However, the notion of false-positive TIM clearly points out that we still have not fully understood all the changes in imaging induced by 1) acute surgery and 2) prior surgery, prior treatment, prior radiotherapy, and recurrent disease. Intraoperative MRI is the most extreme and acute imaging property challenging us with both of these issues. In postoperative MRI, several assessment recommendations exist and have been put forward by working groups such as that of Macdonald et al. ${ }^{27}$ or the Response Assessment in Neuro-Oncology group to evaluate the effectiveness of clinical therapy and surgery in brain tumors. ${ }^{28,29}$ However, these criteria have been adjusted to postoperative timing (24-72 hours after surgery), and ioMRI unapologetically eliminates this timeframe by being directly acquired from the operating room. ${ }^{28}$ The future challenge in the field of ioMRI will be to develop standardized criteria and imaging protocols to not only overcome the challenges of interpretation of ioMRI but also emphasize the surgical benefit of ioMRI for our patients.

\section{Conclusions}

The differentiation and identification of tumor remnants in surgery of recurrent tumor lesions is more challenging. There was evidence for a significantly increased incidence of TIM in the re-craniotomy group in comparison with the primary craniotomy group in this study. Most of these false-positive signals were contrast-enhancing signals, which in situ turned out to be hemorrhages. Hyperacute hemorrhage is difficult to distinguish in MRI sequences based on contrast enhancement. The usage of an SWI sequence for differentiation between residual tumor and hemorrhage during ioMRI could be advisable.

\section{References}

1. Bloch O, Han SJ, Cha S, et al. Impact of extent of resection for recurrent glioblastoma on overall survival: clinical article. J Neurosurg. 2012;117(6):1032-1038.

2. Lacroix M, Abi-Said D, Fourney DR, et al. A multivariate analysis of 416 patients with glioblastoma multiforme: prognosis, extent of resection, and survival. J Neurosurg. 2001; 95(2):190-198.

3. Scherer M, Ahmeti H, Roder C, et al. Surgery for diffuse WHO grade II gliomas: volumetric analysis of a multicenter retrospective cohort from the German Study Group for Intraoperative Magnetic Resonance Imaging. Neurosurgery. 2020; 86(1):E64-E74.

4. Schneider JP, Trantakis C, Rubach M, et al. Intraoperative MRI to guide the resection of primary supratentorial glioblastoma multiforme-a quantitative radiological analysis. Neuroradiology. 2005;47(7):489-500.

5. Suchorska B, Weller M, Tabatabai G, et al. Complete resection of contrast-enhancing tumor volume is associated with improved survival in recurrent glioblastoma-results from the DIRECTOR trial. Neuro Oncol. 2016;18(4):549-556.

6. Kubben PL, ter Meulen KJ, Schijns OE, et al. Intraoperative MRI-guided resection of glioblastoma multiforme: a systematic review. Lancet Oncol. 2011;12(11):1062-1070.

7. Nimsky C, Ganslandt O, Cerny S, et al. Quantification of, visualization of, and compensation for brain shift using intraoperative magnetic resonance imaging. Neurosurgery. 2000; 47(5):1070-1080.

8. Nabavi A, Black PM, Gering DT, et al. Serial intraoperative magnetic resonance imaging of brain shift. Neurosurgery. 2001;48(4):787-798.

9. Wirtz CR, Knauth M, Staubert A, et al. Clinical evaluation and follow-up results for intraoperative magnetic resonance imaging in neurosurgery. Neurosurgery. 2000;46(5):1112_ 1122.

10. Senft C, Bink A, Franz K, et al. Intraoperative MRI guidance and extent of resection in glioma surgery: a randomised, controlled trial. Lancet Oncol. 2011;12(11):997-1003.

11. Claus EB, Horlacher A, Hsu L, et al. Survival rates in patients with low-grade glioma after intraoperative magnetic resonance image guidance. Cancer. 2005;103(6):1227-1233.

12. Nabavi A, Thurm H, Zountsas B, et al. Five-aminolevulinic acid for fluorescence-guided resection of recurrent malignant gliomas: a phase II study. Neurosurgery. 2009;65(6):10701077.

13. Hammoud MA, Ligon BL, elSouki R, et al. Use of intraop- 
erative ultrasound for localizing tumors and determining the extent of resection: a comparative study with magnetic resonance imaging. J Neurosurg. 1996;84(5):737-741.

14. Dinevski N, Sarnthein J, Vasella F, et al. Postoperative neurosurgical infection rates after shared-resource intraoperative magnetic resonance imaging: a single-center experience with 195 cases. World Neurosurg. 2017;103:275-282.

15. Bohinski RJ, Kokkino AK, Warnick RE, et al. Glioma resection in a shared-resource magnetic resonance operating room after optimal image-guided frameless stereotactic resection. Neurosurgery. 2001;48(4):731-744.

16. Reifenberger G, Wirsching HG, Knobbe-Thomsen CB, Weller M. Advances in the molecular genetics of gliomasimplications for classification and therapy. Nat Rev Clin Oncol. 2017;14(7):434-452.

17. Nimsky C, Ganslandt O, Von Keller B, et al. Intraoperative high-field-strength MR imaging: implementation and experience in 200 patients. Radiology. 2004;233(1):67-78.

18. Hatiboglu MA, Weinberg JS, Suki D, et al. Impact of intraoperative high-field magnetic resonance imaging guidance on glioma surgery: a prospective volumetric analysis. Neurosurgery. 2009;64(6):1073-1081.

19. Kuhnt D, Becker A, Ganslandt O, et al. Correlation of the extent of tumor volume resection and patient survival in surgery of glioblastoma multiforme with high-field intraoperative MRI guidance. Neuro Oncol. 2011;13(12):1339-1348.

20. Coburger J, Engelke J, Scheuerle A, et al. Tumor detection with 5-aminolevulinic acid fluorescence and Gd-DTPA-enhanced intraoperative MRI at the border of contrast-enhancing lesions: a prospective study based on histopathological assessment. Neurosurg Focus. 2014;36(2):E3.

21. Coburger J, Merkel A, Scherer M, et al. Low-grade glioma surgery in intraoperative magnetic resonance imaging: results of a multicenter retrospective assessment of the German Study Group for Intraoperative Magnetic Resonance Imaging. Neurosurgery. 2016;78(6):775-786.

22. Quick-Weller J, Lescher S, Forster MT, et al. Combination of 5-ALA and iMRI in re-resection of recurrent glioblastoma. Br J Neurosurg. 2016;30(3):313-317.

23. Gustafsson O, Rossitti S, Ericsson A, Raininko R. MR imaging of experimentally induced intracranial hemorrhage in rabbits during the first 6 hours. Acta Radiol. 1999;40(4): $360-368$
24. Halefoglu AM, Yousem DM. Susceptibility weighted imaging: clinical applications and future directions. World J Radiol. 2018;10(4):30-45.

25. Rohde V, Rohde I, Thiex R, et al. The role of intraoperative magnetic resonance imaging for the detection of hemorrhagic complications during surgery for intracerebral lesions an experimental approach. Surg Neurol. 2001;56(4):266-275.

26. Giordano M, Samii A, Lawson McLean AC, et al. Intraoperative magnetic resonance imaging in pediatric neurosurgery: safety and utility. J Neurosurg Pediatr. 2017;19(1):77-84.

27. Macdonald DR, Cascino TL, Schold SC Jr, Cairncross JG. Response criteria for phase II studies of supratentorial malignant glioma. J Clin Oncol. 1990;8(7):1277-1280.

28. Vogelbaum MA, Jost S, Aghi MK, et al. Application of novel response/progression measures for surgically delivered therapies for gliomas: Response Assessment in Neuro-Oncology (RANO) Working Group. Neurosurgery. 2012;70(1):234-244.

29. van den Bent MJ, Vogelbaum MA, Wen PY, et al. End point assessment in gliomas: novel treatments limit usefulness of classical Macdonald's criteria. J Clin Oncol. 2009;27(18): 2905-2908.

\section{Disclosures}

The authors report no conflict of interest concerning the materials or methods used in this study or the findings specified in this paper.

\section{Author Contributions}

Conception and design: Wang, Bozinov. Acquisition of data: Wang, Selge. Analysis and interpretation of data: Wang, Scheffler. Drafting the article: Wang. Critically revising the article: Sebök, Yang, Brandi, Winklhofer. Reviewed submitted version of manuscript: Bozinov. Statistical analysis: Wang, Selge. Study supervision: Bozinov.

\section{Correspondence}

Sophie S. Wang: Eberhard Karls University Tübingen, Germany. sophie.wang@med.uni-tuebingen.de. 